

\title{
Proof of the resolution-doubling of Random Illumination Microscopy using the variance of the speckled images
}

\author{
Simon Labouesse, Jérôme Idier, Anne Sentenac, Marc Allain, Thomas
}

\author{
Mangeat
}

\section{- To cite this version:}

Simon Labouesse, Jérôme Idier, Anne Sentenac, Marc Allain, Thomas Mangeat. Proof of the resolution-doubling of Random Illumination Microscopy using the variance of the speckled images. 2021 29th European Signal Processing Conference (EUSIPCO), Aug 2021, Dublin, Ireland. pp.1159$1162,10.23919 /$ EUSIPCO54536.2021.9616038 . hal-03538553

\section{HAL Id: hal-03538553 \\ https://hal.science/hal-03538553}

Submitted on 21 Jan 2022

HAL is a multi-disciplinary open access archive for the deposit and dissemination of scientific research documents, whether they are published or not. The documents may come from teaching and research institutions in France or abroad, or from public or private research centers.
L'archive ouverte pluridisciplinaire HAL, est destinée au dépôt et à la diffusion de documents scientifiques de niveau recherche, publiés ou non, émanant des établissements d'enseignement et de recherche français ou étrangers, des laboratoires publics ou privés. 


\section{Proof of the resolution-doubling of Random Illumination Microscopy using the variance of the speckled images}

\author{
$1^{\text {st }}$ Simon Labouesse \\ Aix Marseille Université \& CNRS, IBDM - UMR7288 \\ \& Turing Centre for Living Systems \\ Campus de Luminy Case 907, 13288 Marseille, France \\ simon.labouesse@gmail.com \\ $3^{\text {rd }}$ Anne Sentenac \\ Aix Marseille University, CNRS, Centrale Marseille \\ Institut Fresnel, F-13013, Marseille, France \\ anne.sentenac@fresnel.fr \\ $5^{\text {th }}$ Thomas Mangeat \\ Centre for Integrative Biology, Université de Toulouse \\ CNRS, UPS, F-31062 Toulouse \\ thomas.mangeat@univ-tlse3.fr
}

\author{
$2^{\text {nd }}$ Jérôme Idier \\ Laboratoire des Sciences du Numérique de Nantes \\ Centrale Nantes, F-44321 Nantes, France \\ jerome.idier@1s2n.fr
}

\author{
$4^{\text {th }}$ Marc Allain \\ Aix Marseille University, CNRS, Centrale Marseille \\ Institut Fresnel, F-13013, Marseille, France \\ marc.allain@fresnel.fr
}

\begin{abstract}
Owing to minor modifications of the optical setup, Random Illumination Microscopy (RIM) surpasses the resolution limit of a standard fluorescence microscope. RIM uses speckle illuminations of the sample to derive a single variance image from the resulting diffraction-limited acquisitions. Variancematching iterations then produce a super-resolved estimate of the sample. Here, we demonstrate that in the noiseless case, variancematching yields a unique solution for the set of spatial frequencies corresponding to a doubled resolution limit. A similar result was already proven for covariance-matching, but covariancebased iterations are not implementable in practice, due to the huge size of the covariance matrix and to the induced numerical complexity. Our new identifiability result is a strong theoretical evidence supporting the super-resolution capability of the variance-matching version of RIM.
\end{abstract}

Index Terms-Computational imaging, Quadratic inverse problems, Multi-illumination imaging, Super-resolution, Cutoff frequency, Second-order statistics, Variance, Optical microscopy

\section{INTRODUCTION}

Fluorescence microscopy is an indispensable tool in cell biology but its lateral resolution, limited by diffraction to about $300 \mathrm{~nm}$ for the best set-ups, remains generally insufficient for following the dynamic interplay of the cell constituents. Several super-resolution (SR) microscopy approaches have been proposed in the last twenty years [1] to tackle this issue, among which the best compromise between high resolution and practical in-vivo imaging is Structured Illumination Microscopy (SIM) [2], [3]. SIM consists in recording several

The authors acknowledge partial financial support for this paper from the Agence Nationale de la Recherche under Grant ANR-12-BS03-0006 and the ITMO Cancer of the 'Plan Cancer 2014-2019'. low-resolution images of the sample under different positions and orientations of a known periodic illumination. The role of the periodic illumination is to down-modulate previously inaccessible sample high spatial frequencies into the Fourier support of the observation Point Spread Function (PSF). After some straightforward manipulations, a correspondence can be found between SIM low-resolution images and the spatial frequencies of the sample over an enlarged Fourier support which can reach twice that of a widefield fluorescence microscope. Yet, this identifiability requires the knowledge and thus a tight control of the illumination patterns which limits SIM application to weakly scattering samples and complexifies the experimental set-up [4].

In order to release this major constraint, it has been proposed to replace the periodic illumination of SIM by random uncontrolled speckles [5], [6]. A speckle is the light pattern formed when a coherent beam (laser) is reflected or transmitted by a random diffusive medium. The key property of random speckle illumination is that its statistics (average and covariance) are perfectly known and, furthermore, insensitive to aberrations and scattering. Random Illumination Microscopy (RIM) is thus expected to be easier to use than SIM, as it does not require the control of the illumination, with an extended application domain, as it is insensitive to aberrations on the excitation side. Yet, contrary to SIM, the ability to extract the spatial frequencies of the sample over an enlarged Fourier support from the low-resolution speckled images of RIM is not a straightforward issue.

Recently, we have shown mathematically that, provided the frequency support of the speckle patterns is similar to that of 
the PSF, the theoretical SR capacity of RIM is identical to that of SIM [7]. Our demonstration exploited the statistical covariance matrix of the recorded speckled images, which was shown to depend quadratically on the sample fluorescence and on the known speckle covariance and PSF. Yet, although theoretically appealing, this approach could not be transformed into a realistic numerical scheme as images of $1000 \times 1000$ pixels generate a covariance matrix of size $10^{6} \times 10^{6}$ which is not tractable on modern computer systems.

In this letter, we prove a new result about RIM that reconciles the theoretical SR capacity and an affordable computational burden. Indeed, under fairly realistic assumptions, we mathematically show that the statistical variance of the recorded images is sufficient to recover an image of the sample with the same SR factor as covariance-based RIM.

Second-order statistics being quadratic functions of the unknown sample, the issue of retrieving spatial frequency components of the latter from either the variance or the covariance of the speckle images belongs to the family of quadratic inverse problems (QIP) [8]. As a consequence, it is substantially more difficult to formally characterize the SR capacity of RIM, compared to SIM.

Let us also mention that variance-based microscopy using speckle illuminations has been already proposed, either for its sectioning properties [9], or in the context of superresolution [10]. However, to our best knowledge, our contribution is the first one to mathematically characterize the super-resolution property of variance-based RIM.

In Section II, we introduce a mathematical description of the image model. For sake of self-consistency, we also recall known results for covariance-based RIM. Our novel result concerning variance-based RIM is presented in Section III, and elements of discussion are given in Section IV.

\section{PhysicAl ASSUMPTIONS AND KNOWN RESUltS}

\section{A. Imaging model}

For the sake of clarity, we restrict ourselves to the case of two-dimensional samples, and we formulate the problem in a fully discrete setting, where both the recorded images and the sample are represented on fine grids, with a sampling rate common to both. RIM images can then be modelled by:

$$
\boldsymbol{z}_{m}=\boldsymbol{y}_{m}+\boldsymbol{\epsilon}_{m}
$$

with

$$
\boldsymbol{y}_{m}=\boldsymbol{H}\left(\boldsymbol{\rho} \circ \boldsymbol{I}_{m}\right),
$$

where $\epsilon_{m}$ is a random variable modeling an additive noise, $\boldsymbol{y}_{m}$ is a vectorized image corresponding to the $m$ th speckle illumination $\boldsymbol{I}_{m}, \boldsymbol{H}$ a convolution matrix corresponding to a convolution by the Point Spread Function (PSF) $\boldsymbol{h}$ of the microscope, $\rho$ the fluorescence density map to recover, and $\circ$ the Hadamard (i.e., entrywise, or Schur) matrix product [11, Chapter 5].
When the speckle illuminations are fully developed, the speckle covariance $\operatorname{Cov}\left(\boldsymbol{I}_{m}\right)=\boldsymbol{C}$ is a known convolution operator [12]. Then, the covariance matrix of $\boldsymbol{z}_{m}$ reads

$$
\Gamma_{z}(\rho)=\Gamma_{y}(\rho)+\Gamma_{\epsilon}
$$

with

$$
\Gamma_{y}(\rho)=H \operatorname{Diag}(\rho) C \operatorname{Diag}(\rho) H^{\mathrm{t}} .
$$

The variance identifies with the diagonal of the covariance matrix $\boldsymbol{v}_{\boldsymbol{z}}(\boldsymbol{\rho})=\operatorname{diag}\left(\boldsymbol{\Gamma}_{\boldsymbol{z}}\right)$. Assuming the noise covariance $\operatorname{Cov}\left(\boldsymbol{\epsilon}_{m}\right)=\boldsymbol{\Gamma}_{\boldsymbol{\epsilon}}$ is known, the knowledge of $\boldsymbol{v}_{\boldsymbol{z}}$ is thus equivalent to that of

$$
\boldsymbol{v}_{\boldsymbol{y}}=\operatorname{diag}\left(\boldsymbol{\Gamma}_{\boldsymbol{y}}\right)
$$

Hereafter, we refer to $\boldsymbol{v}_{\boldsymbol{y}}$ and $\boldsymbol{\Gamma}_{\boldsymbol{y}}$ as $\boldsymbol{v}$ and $\boldsymbol{\Gamma}$, respectively.

In this document, we assume that the illumination and observation of the sample are performed through a perfect optical mounting so that $\boldsymbol{H}$ and $\boldsymbol{C}$ are nonnegative definite convolution operators defined by the Airy functions $\boldsymbol{h}$ and $c$ [13, Sec. 4.4.2] with frequency cut-off $f_{\mathrm{PSF}}=2 \mathrm{NA}_{\mathrm{obs}} / \lambda_{\mathrm{obs}}$ and $f_{\text {spec }}=2 \mathrm{NA}_{\text {ill }} / \lambda_{\text {ill }}$ respectively, with $\mathrm{NA}_{\text {obs(ill })}$ the numerical aperture of the observation (illumination) objective and $\lambda_{\text {obs(ill) }}$ the emission (excitation) wavelength.

Since our goal is to demonstrate a factor two in terms of super-resolution, the sampling rate of the object must be at least four times the cutoff frequency imposed by the PSF. In the rest of this document, we make use of the following notations: $f_{\mathrm{PSF}} \leq 1 / 4$ denotes the normalized cutoff frequency imposed by the PSF, and

$$
\mathcal{G}=\left\{\boldsymbol{\nu} \in \mathbb{R}^{d},\|\boldsymbol{\nu}\|_{\infty}<1 / 2\right\} \cap\left\{\boldsymbol{n} / N, \boldsymbol{n} \in \mathbb{Z}^{d}\right\}
$$

denotes the $d$-dimensional normalized frequency grid limited by the Nyquist frequency ( $d=2$ for $2 \mathrm{D}$ imaging). Here, we assume that RIM acquisitions $\boldsymbol{z}_{m}$ are made of $N=n^{d}$ elements. Then each of them can be decomposed over the set of discrete frequencies $\mathcal{D}_{\mathrm{PSF}}=\mathcal{D}\left(f_{\mathrm{PSF}}\right)$, where $\mathcal{D}(f)$ is a generic notation for the "discrete interior" of a ball of radius $f$ :

$$
\mathcal{D}(f)=\{\boldsymbol{\nu} \in \mathcal{G},\|\boldsymbol{\nu}\|<f\} .
$$

\section{B. Covariance-based RIM}

In the 2D case, [7] obtains that the knowledge of $\Gamma$ allows to retrieve the frequency components of $\rho$ within the ball $\mathcal{D}_{\mathrm{SR}}=$ $\mathcal{D}\left(2 f_{\text {spec }}\right)$, provided that $f_{\text {spec }} \leq f_{\mathrm{PSF}}$. When $f_{\mathrm{spec}}=f_{\mathrm{PSF}}$, we have $\mathcal{D}_{\mathrm{SR}}=\mathcal{D}\left(2 f_{\mathrm{PSF}}\right)$, which exactly corresponds to an $\mathrm{SR}$ factor equal to two.

Theorem 1. Let $\rho$ be any entrywise nonnegative vector of size $N$. For any entrywise nonnegative solution $q$ to the quadratic system $\boldsymbol{\Gamma}(\boldsymbol{q})=\boldsymbol{\Gamma}(\boldsymbol{\rho})$, the frequency components of $\boldsymbol{q}$ coincide with that of $\boldsymbol{\rho}$ in $\mathcal{D}_{\mathrm{SR}}$.

Proof: The fact that all frequency components in $\mathcal{D}_{\mathrm{SR}}$ can be retrieved from $\Gamma(\rho)$ is based on a unicity argument for the factorization. We recall that any nonnegative definite operator admits a unique, well-defined square root matrix and that $\boldsymbol{H}$ and $\boldsymbol{C}$ are nonnegative definite convolution operators 
while $\boldsymbol{\Gamma}(\boldsymbol{\rho})$ is a nonnegative definite operator. The covariance $\Gamma(\rho)$ can be cast as

$$
\begin{aligned}
\Gamma(\rho) & =H \operatorname{Diag}(\rho) C \operatorname{Diag}(\rho) H \\
& =H \operatorname{Diag}(\rho) \sqrt{C} \sqrt{C} \operatorname{Diag}(\rho) H .
\end{aligned}
$$

If $f_{\mathrm{spec}} \leq f_{\mathrm{PSF}}$, one can find a nonnegative convolution operator $\boldsymbol{A}$ such that $\boldsymbol{A H}=\boldsymbol{H} \boldsymbol{A}=\sqrt{\boldsymbol{C}}$. Then, we can identify the square root of $A \Gamma(\rho) A$ to $\sqrt{C} \operatorname{Diag}(\rho) \sqrt{C}$. Noting that $\operatorname{diag}(\sqrt{C} \operatorname{Diag}(\rho) \sqrt{C})=c^{2} \star \rho$ where $\star$ is the convolution product, we conclude that the knowledge of $\boldsymbol{\Gamma}(\boldsymbol{\rho})$ uniquely determines the spectral components of $\rho$ in $\mathcal{D}_{\mathrm{SR}}$.

We also stress that this result is tight when $f_{\mathrm{spec}}=f_{\mathrm{PSF}}$ (and in particular in the case $\boldsymbol{H}=\boldsymbol{C}$ ) since the frequency components of $\boldsymbol{q}$ outside $\mathcal{D}\left(2 f_{\mathrm{PSF}}\right)$ are not identifiable, according to the following proposition.

Proposition 1. Let $\boldsymbol{\rho}$ be any vector of size $N$. Then $\boldsymbol{\Gamma}(\boldsymbol{\rho}+\boldsymbol{\delta})=$ $\boldsymbol{\Gamma}(\boldsymbol{\rho})$ for any vector $\boldsymbol{\delta}$ with no components in $\mathcal{D}\left(f_{\mathrm{PSF}}+f_{\mathrm{spec}}\right)$.

Proof: For any vector $\boldsymbol{\delta}$ with no components in $\mathcal{D}\left(f_{\mathrm{PSF}}+\right.$ $\left.f_{\text {spec }}\right)$, each column of matrix $\operatorname{Diag}(\boldsymbol{\delta}) \boldsymbol{C}$ has no frequency components in $\mathcal{D}_{\mathrm{PSF}}$, so $\boldsymbol{H} \operatorname{Diag}(\boldsymbol{\delta}) \boldsymbol{C}=\boldsymbol{C D i a g}(\boldsymbol{\delta}) \boldsymbol{H}=\mathbf{0}$. As a consequence,

$$
\begin{aligned}
\Gamma(\rho+\delta) & =H \operatorname{Diag}(\rho+\delta) C \operatorname{Diag}(\rho+\delta) H \\
& =\Gamma(\rho)+\Gamma(\delta)+H \operatorname{Diag}(\rho) C \operatorname{Diag}(\delta) H \\
& +H \operatorname{Diag}(\delta) C \operatorname{Diag}(\rho) H \\
& =\Gamma(\rho) .
\end{aligned}
$$

\section{VARIANCE-BASED RIM}

\section{A. Super-resolution from variance equations}

The quadratic system of Theorem 1 is made of $\frac{1}{2} N(N+1)$ real equations, for only $M$ free real-valued variables, where $M$ stand for the cardinality of $\mathcal{D}_{\mathrm{SR}}$. Since $M \leq \frac{\pi}{4} N$ in 2D (and $M \leq N$ in 1D), there is room left for a refined identifiability result, using a smaller number of equations.

Here, we show that the $N$ variance equations are sufficient to uniquely determine the $M$ frequency components in $\mathcal{D}_{\mathrm{SR}}$, provided that $\rho$ is an entrywise positive vector and that $\boldsymbol{H}=\boldsymbol{C}$. This last condition is satisfied if the illumination and observation are performed through the same objective and that the Stokes shift is neglected.

This important result is easily deduced from two technical results, Lemmas 1 and 2, which we derive first.

Let us define

$$
\begin{aligned}
M_{x} & =H \operatorname{Diag}(x) H, \\
B_{x} & =H \circ M_{x},
\end{aligned}
$$

In particular, for a given object $\rho$, the (noiseless) data variance vector (5) is given by $\boldsymbol{v}(\boldsymbol{\rho})=\boldsymbol{B}_{\boldsymbol{\rho}} \boldsymbol{\rho}$ according to the matrix identity [14]:

$$
\operatorname{diag}\left(\mathbf{A D i a g}(\boldsymbol{x}) \mathbf{B}^{\mathrm{t}}\right)=(\mathbf{A} \circ \mathbf{B}) \boldsymbol{x}=(\mathbf{B} \circ \mathbf{A}) \boldsymbol{x} .
$$

Lemma 1. For any two real solutions $\boldsymbol{\rho}$ and $\boldsymbol{q}$ to Eq. (5), we have $\boldsymbol{\rho}-\boldsymbol{q} \in \operatorname{Ker}\left(\boldsymbol{B}_{\boldsymbol{\rho}+\boldsymbol{q}}\right)$ and $\boldsymbol{\rho}+\boldsymbol{q} \in \operatorname{Ker}\left(\boldsymbol{B}_{\boldsymbol{\rho}-\boldsymbol{q}}\right)$.

Proof: Let us define the bilinear vector-valued function:

$$
\boldsymbol{f}(\boldsymbol{x}, \boldsymbol{y})=\operatorname{diag}(H \operatorname{Diag}(\boldsymbol{x}) H \operatorname{Diag}(y) H),
$$

so that

$$
\boldsymbol{f}(\boldsymbol{x}, \boldsymbol{y})=\boldsymbol{B}_{\boldsymbol{x}} \boldsymbol{y}=\boldsymbol{B}_{\boldsymbol{y}} \boldsymbol{x}
$$

and $\boldsymbol{v}(\boldsymbol{\rho})=\boldsymbol{f}(\boldsymbol{\rho}, \boldsymbol{\rho})$. Each component of $\boldsymbol{f}$ is a symmetric form, since $\boldsymbol{f}(\boldsymbol{x}, \boldsymbol{y})=\boldsymbol{f}(\boldsymbol{y}, \boldsymbol{x})$.

Indeed,

$$
\begin{aligned}
\boldsymbol{f}(\boldsymbol{\rho}+\boldsymbol{q}, \boldsymbol{\rho}-\boldsymbol{q}) & =\boldsymbol{f}(\boldsymbol{\rho}, \boldsymbol{\rho})-\boldsymbol{f}(\boldsymbol{q}, \boldsymbol{q})+\boldsymbol{f}(\boldsymbol{q}, \boldsymbol{\rho})-\boldsymbol{f}(\boldsymbol{\rho}, \boldsymbol{q}) \\
& =\boldsymbol{v}-\boldsymbol{v}+\boldsymbol{f}(\boldsymbol{q}, \boldsymbol{\rho})-\boldsymbol{f}(\boldsymbol{q}, \boldsymbol{\rho}) \\
& =\mathbf{0} .
\end{aligned}
$$

Combining Equations (10) and (11), we obtain

$$
B_{\rho+q}(\rho-q)=B_{\rho-q}(\rho+q)=0,
$$

which proves the assertion.

Lemma 2. For any vector $\boldsymbol{x}$ with positive entries, $\operatorname{Ker}\left(\boldsymbol{B}_{\boldsymbol{x}}\right)$ is the linear span of frequency components outside $\mathcal{D}_{\mathrm{SR}}$.

Proof: Let $K_{\min }=\min (\boldsymbol{x})$, so that $\boldsymbol{x}_{\min }=\boldsymbol{x}-K_{\min }$ is entrywise nonnegative. We have $\boldsymbol{B}_{\boldsymbol{x}}=K_{\min } \boldsymbol{G}+\boldsymbol{B}_{\boldsymbol{x}_{\min }}$, with $\boldsymbol{G}=\boldsymbol{H}^{2} \circ \boldsymbol{H}$. For all $\boldsymbol{z} \in \operatorname{Ker}\left(\boldsymbol{B}_{\boldsymbol{x}}\right)$, we have $\boldsymbol{z}^{\mathrm{t}} \boldsymbol{B}_{\boldsymbol{x}} \boldsymbol{z}=\boldsymbol{z}^{\mathrm{t}} \boldsymbol{G} \boldsymbol{z}+\boldsymbol{z}^{\mathrm{t}} \boldsymbol{B}_{\boldsymbol{x}_{\min }} \boldsymbol{z}=0$. Now, the matrices $\boldsymbol{G}$ and $\boldsymbol{B}_{\boldsymbol{x}_{\min }}$, which are the Hadamard product between two nonnegative definite matrices, are also nonnegative definite according to the Schur product theorem [11, Theorem 5.2.1]. Therefore, $\operatorname{Ker}\left(\boldsymbol{B}_{\boldsymbol{x}}\right)=\operatorname{Ker}(\boldsymbol{G}) \cap \operatorname{Ker}\left(\boldsymbol{B}_{\boldsymbol{x}_{\min }}\right) \subset \operatorname{Ker}(\boldsymbol{G})$.

Similarly, let $K_{\max }=\max (\boldsymbol{x})$, so that $\boldsymbol{x}_{\max }=K_{\max }-\boldsymbol{x}$ is entrywise nonnegative. We have $\boldsymbol{B}_{\boldsymbol{x}}=K_{\max } \boldsymbol{G}-\boldsymbol{B}_{\boldsymbol{x}_{\max }}$, and $\boldsymbol{B}_{\boldsymbol{x}_{\max }}$ and $\boldsymbol{B}_{\boldsymbol{x}}$ are both nonnegative definite. For all $\boldsymbol{z} \in \operatorname{Ker}(\boldsymbol{G}), \boldsymbol{z}^{\mathrm{t}} \boldsymbol{B}_{\boldsymbol{x}} \boldsymbol{z}=-\boldsymbol{z}^{\mathrm{t}} \boldsymbol{B}_{\boldsymbol{x}_{\max }} \boldsymbol{z}$, where the lhs and the rhs are nonnegative and nonpositive, respectively. We conclude that $\boldsymbol{z}^{\mathrm{t}} \boldsymbol{B}_{\boldsymbol{x}} \boldsymbol{z}=0$, so $\operatorname{Ker}(\boldsymbol{G}) \subset \operatorname{Ker}\left(\boldsymbol{B}_{\boldsymbol{x}}\right)$. Recalling that $\operatorname{Ker}\left(\boldsymbol{B}_{\boldsymbol{x}}\right) \subset \operatorname{Ker}(\boldsymbol{G})$ we have demonstrated that $\operatorname{Ker}\left(\boldsymbol{B}_{\boldsymbol{x}}\right)=$ $\operatorname{Ker}(\boldsymbol{G})$. Now, matrix $\boldsymbol{G}$ is a convolution operator with kernel $\boldsymbol{g}=(\boldsymbol{h} \star \boldsymbol{h}) \circ \boldsymbol{h}$, with $\widetilde{\boldsymbol{g}}=(\widetilde{\boldsymbol{h}} \odot \widetilde{\boldsymbol{h}}) \star \widetilde{\boldsymbol{h}}$ the Fourier transform of $\boldsymbol{g}$. The filter $\widetilde{\boldsymbol{g}}$ has nonzero components for all spatial frequencies belonging to $\mathcal{D}_{\mathrm{SR}}$. The $\operatorname{kernel} \operatorname{Ker}(\boldsymbol{G})$ is the linear span of frequency components outside $\mathcal{D}_{\mathrm{SR}}$.

Finally, let us consider two entrywise positive solutions $\rho$ and $\boldsymbol{q}$ to Eq. (5). According to Lemma 1, we have $\boldsymbol{\rho}-\boldsymbol{q} \in$ $\operatorname{Ker}\left(\boldsymbol{B}_{\boldsymbol{\rho}+\boldsymbol{q}}\right)$, where $\boldsymbol{\rho}+\boldsymbol{q}$ is entrywise positive. Therefore, we can conclude from Lemma 2 that $\boldsymbol{\rho}-\boldsymbol{q}$ is in the linear span of frequency components outside $\mathcal{D}_{\mathrm{SR}}$. Moreover, we know from Proposition 1 that the frequency components of $\boldsymbol{q}$ outside $\mathcal{D}_{\mathrm{SR}}$ have no impact on the data covariance, and hence on its diagonal. These results are summarized in the following theorem, providing the new identification result we aimed at.

Theorem 2. Let $\rho$ be any entrywise positive vector of size $N$. For any entrywise nonnegative solution $\boldsymbol{q}$ to the quadratic system of $N$ equations $\boldsymbol{v}(\boldsymbol{q})=\boldsymbol{v}(\boldsymbol{\rho})$, the frequency components of $\boldsymbol{q}$ coincide with that of $\boldsymbol{\rho}$ in $\mathcal{D}_{\mathrm{SR}}$, while the frequency 
components of $\boldsymbol{q}$ outside $\mathcal{D}_{\mathrm{SR}}$ remain arbitrary (up to the nonnegativity constraint on the entries of $q$ ).

Several comments can be made about this variance-based result, compared to its covariance-based counterpart. First, we stress that Theorem 1 holds if $f_{\mathrm{PSF}}=f_{\mathrm{spec}}$ whereas Theorem 2 requires that the matrices $\boldsymbol{H}$ and $\boldsymbol{C}$ are identical (for the matrix $\boldsymbol{B}_{\boldsymbol{x}}$ to be nonnegative definite) This condition is more stringent. Actually, we have found a small size counterexample with $f_{\mathrm{PSF}}=f_{\mathrm{spec}}$, and where the $N$ variance equations admit two distinct entrywise positive solutions. This indicates that the assumptions of Theorem 2 are somewhat tight. A natural perspective would be to investigate if the identification result could be retrieved with $\boldsymbol{H} \neq \boldsymbol{C}$ thanks to short-range correlations added to the variance.Note however that, if one has access to the raw images given by Eq. (2), it is always possible to filter the images such that $\boldsymbol{H}$ becomes $\boldsymbol{C}$ provided $f_{\mathrm{PSF}} \geq f_{\mathrm{spec}}$.

Another important difference between Theorems 1 and 2 concerns the fact that strict positivity of the sample is needed in the latter case. We have some preliminary results showing that this condition could be relaxed, but the maximal number of zero entries allowing a provable super-resolution is currently indeterminate.

\section{CONCLUSION}

This paper provides a mathematical proof that the superresolution capacity of random illumination microscopy still holds when only the statistical variance of the recorded lowresolution speckled images is considered instead of the full covariance. Such a theoretical result meets practical evidences recently obtained concerning $2 \mathrm{D}$ variance-based imaging applied to various types of biological samples [15].

As an important final remark, we note that a formal extension of our new identification result to the three-dimensional (3D) case is straightforward, with the benefit of an axial superresolution effect, on top of the lateral one obtained in 2D. Real-world applications would consist in recording multiple speckled images of the sample at different depths. This can be done in a sequential manner, by translating the sample through the focal plane, or simultaneously using a multifocus system [16]).

\section{REFERENCES}

[1] L. Schermelleh, A. Ferrand, T. Huser, C. Eggeling, M. Sauer, O. Biehlmaier, and G. P. Drummen, "Super-resolution microscopy demystified," Nature cell biology, vol. 21, no. 1, pp. 72-84, 2019.

[2] R. Heintzmann and C. G. Cremer, "Laterally modulated excitation microscopy: improvement of resolution by using a diffraction grating," Proc. SPIE, vol. 3568, pp. 185-196, 1999. [Online]. Available: http://dx.doi.org/10.1117/12.336833

[3] M. G. L. Gustafsson, D. A. Agard, and J. W. Sedat, "Doubling the lateral resolution of wide-field fluorescence microscopy using structured illumination," Proc. SPIE, vol. 3919, pp. 141-150, 2000. [Online]. Available: http://dx.doi.org/10.1117/12.384189

[4] J. Demmerle, C. Innocent, A. J. North, G. Ball, M. M. 3, E. Miron, A. Matsuda, I. M. Dobbie, Y. Markaki, and L. Schermelleh, "Strategic and practical guidelines for successful structured illumination microscopy," Nature Protocols, vol. 12, no. 5, pp. 988-1010, 2017.
[5] E. Mudry, K. Belkebir, J. Girard, J. Savatier, E. Le Moal, C. Nicoletti, M. Allain, and A. Sentenac, "Structured illumination microscopy using unknown speckle patterns," Nature Photonics, vol. 6, no. 5, pp. 312-315, 2012.

[6] S. Labouesse, A. Negash, J. Idier, S. Bourguignon, T. Mangeat, P. Liu, A. Sentenac, and M. Allain, "Joint reconstruction strategy for structured illumination microscopy with unknown illuminations," vol. 26, no. 5, pp. 1-14, May 2017.

[7] J. Idier, S. Labouesse, P. Liu, M. Allain, S. Bourguignon, and A. Sentenac, "On the super-resolution capacity of imagers using unknown speckle illuminations," vol. 4, no. 1, pp. 87-98, Mar. 2018.

[8] R. Beinert and K. Bredies, "Non-convex regularization of bilinear and quadratic inverse problems by tensorial lifting," Inverse Problems, vol. 35, no. 1, p. 015002, 2019.

[9] C. Ventalon and J. Mertz, "Quasi-confocal fluorescence sectioning with dynamic speckle illumination," Opt. Lett., vol. 30, no. 24, pp. 3350-3352, Dec 2005. [Online]. Available: http://ol.osa.org/abstract.cfm?URI=ol-30-24-3350

[10] J.-E. Oh, Y.-W. Cho, G. Scarcelli, and Y.-H. Kim, "Sub-Rayleigh imaging via speckle illumination," Opt. Lett., vol. 38, no. 5, pp. 682-684, Mar 2013. [Online]. Available: http://ol.osa.org/abstract.cfm?URI=ol38-5-682

[11] R. Horn and C. Johnson, Matrix analysis. Cambridge University Press, 1991.

[12] J. W. Goodman, Statistical optics. John Wiley \& Sons, 2015.

[13] - Introduction to Fourier Optics, 2nd ed. San Francisco: McGrawHill Physical and Quantum Electronics Series, 1996.

[14] K. B. Petersen and M. S. Pedersen, The Matrix Cookbook. Technical University of Denmark, Nov. 2012.

[15] T. Mangeat, S. Labouesse, M. Allain, R. Poincloux, A. Bouissou, S. Cantaloube, E. Courtais, E. Vega, T. Li, A. Grégoire, C. Rouvière, S. Allard, N. Campo, M. Suzanne, X. Wang, G. Michaux, M. Pinot, R. Le Borgne, S. Tournier, J. Idier, and A. Sentenac, "Super-resolved live-cell imaging using Random Illumination Microscopy," bioRxiv, 2020. [Online]. Available: https://www.biorxiv.org/content/early/2020/01/27/2020.01.27.905083

[16] S. Abrahamsson, J. Chen, B. Hajj, S. Stallinga, A. Y. Katsov, J. Wisniewski, G. Mizuguchi, P. Soule, F. Mueller, C. D. Darzacq, X. Darzacq, C. Wu, C. I. Bargmann, D. A. Agard, M. Dahan, and M. G. L. Gustafsson, "Fast multicolor 3D imaging using aberration-corrected multifocus microscopy," Nature Methods, vol. 10, pp. 60-63, 2013. 